\title{
Nonlinear impulsive differential and integral inequalities with integral jump conditions
}

Jing Shao ${ }^{1 *}$ and Fanwei Meng ${ }^{2}$

"Correspondence:

shaojing99500@163.com

1 Department of Mathematics,

Jining University, Qufu, Shandong

273155, P.R. China

Full list of author information is

available at the end of the article

\section{Springer}

\begin{abstract}
Some new nonlinear impulsive differential inequalities and integral inequalities with integral jump conditions for discontinuous functions are established using the method of successive iteration. These jump conditions at a discontinuous point are related to the integral conditions of the past state, which can be used in the qualitative analysis of the solutions to certain nonlinear impulsive differential systems.
\end{abstract}

MSC: $34 \mathrm{~A} 37 ; 34 \mathrm{~A} 40$

Keywords: integral inequalities; integral jump condition; discontinuous function; impulsive differential system

\section{Introduction}

Impulsive differential equations, that is, differential equations involving impulse effect, appear as a natural description of observed evolution phenomena of several real world problems. Many processes studied in applied sciences are represented by impulsive differential equations. However, the situation is quite different in many physical phenomena that have a sudden change in their states such as mechanical systems with impact, biological systems such as heart beats, blood flows, population dynamics theoretical physics, pharmacokinetics, mathematical economy, chemical technology, electric technology, metallurgy, ecology, industrial robotics, biotechnology processes, and so on (see [1-3] and [4] for details).

In spite of the importance of impulsive differential equations, the development of the theory of impulsive differential equations has been quite slow due to special features possessed by impulsive differential equations in general, such as pulse phenomena, confluence, and loss of autonomy. Among these results, differential inequalities and integral inequalities with impulsive effects play increasingly important roles in the study of quantitative properties of solutions of impulsive differential systems. However, most of these results involving impulsive effects are point-discontinuous, i.e., impulsive effects are added at a sequence of discontinuous points (see [5-12] for details). For example, in 2004, Borysenko [13] considered the following integral inequality with impulsive effect:

$$
u(t) \leq a(t)+\int_{t_{0}}^{t} f(s) u(s) d s+\sum_{t_{0}<t_{i}<t} \alpha_{i} u^{r}\left(t_{i}-0\right)
$$

(c) 2016 Shao and Meng. This article is distributed under the terms of the Creative Commons Attribution 4.0 International License (http://creativecommons.org/licenses/by/4.0/), which permits unrestricted use, distribution, and reproduction in any medium, provided you give appropriate credit to the original author(s) and the source, provide a link to the Creative Commons license, and indicate if changes were made. 
in 2007, Iovane [14] studied the following integral inequalities:

$$
\begin{aligned}
u(t) \leq & a(t)+\int_{t_{0}}^{t} f(s) u(\lambda(s)) d s+\sum_{t_{0}<t_{i}<t} \alpha_{i} u^{r}\left(t_{i}-0\right) \\
u(t) \leq & a(t)+q(t)\left[\int_{t_{0}}^{t} f(s) u(\alpha(s)) d s+\int_{t_{0}}^{t} f(s) \int_{t_{0}}^{s} g(t) u(\tau(t)) d t d s\right. \\
& \left.+\sum_{t_{0}<t_{i}<t} \alpha_{i} u^{r}\left(t_{i}-0\right)\right]
\end{aligned}
$$

in 2011, Wu-Sheng Wang [5] gave the upper bound for the nonlinear inequality

$$
\nu^{p}(t) \leq A_{0}(t)+\frac{p}{p-q} \int_{t_{0}}^{t} f(s) v^{q}(\tau(s)) d s+\sum_{t_{0}<t_{i}<t} \alpha_{i} v^{q}\left(t_{i}-0\right) .
$$

As we know, most of the phenomena occurring in the natural world do not suddenly change, so the impulsive differential equations with integral jump conditions are more accurate than impulsive differential equations with stationary discontinuous points in characterizing the nature. In 2012, based on a well-known result given by Lakshmikantham et al. [1], Thiramanns and Tarboon [15] studied the following impulsive linear differential inequalities:

$$
\left\{\begin{array}{l}
m^{\prime}(t) \leq p(t) m(t)+q(t), \quad t \neq t_{k} \\
m\left(t_{k}^{+}\right) \leq d_{k} m\left(t_{k}\right)+c_{k} \int_{t_{k}-\tau_{k}}^{t_{k}-\tau_{k}} m(s) d s+b_{k}
\end{array}\right.
$$

and gave the upper-bound estimation of the unknown function $m(t)$.

Theorem 1.1 Suppose that $\left(\mathrm{H}_{0}\right)$ and $\left(\mathrm{H}_{1}\right)$ hold. If $p, q \in C\left[\mathbb{R}_{+}, \mathbb{R}\right]$ and for $k=1,2, \ldots, t \geq t_{0}$, the impulsive linear differential inequality (1.1) holds, where $c_{k} ; d_{k} \geq 0,0 \leq \sigma_{k} \leq \tau_{k} \leq t_{k}-$ $t_{k-1}, b_{k}$ are constants. Then

$$
\begin{aligned}
m(t) \leq & \left\{m\left(t_{0}\right) \prod_{t_{0}<t_{k}<t}\left(d_{k} e^{\int_{t_{k-1}}^{t_{k}} p(\tau) d \tau}+c_{k} \int_{t_{k}-\tau_{k}}^{t_{k}-\sigma_{k}} e^{\int_{t_{k-1}}^{s} p(\tau) d \tau} d s\right)\right. \\
& +\sum_{t_{0}<t_{k}<t}\left[\prod_{t_{k}<t_{j}<t}\left(d_{j} e^{\int_{t_{j-1}}^{t_{j}} p(\tau) d \tau}+c_{j} \int_{t_{j}-\tau_{j}}^{t_{j}-\sigma_{j}} e^{\int_{t_{j-1}}^{s} p(\tau) d \tau} d s\right)\right. \\
& \times\left(d_{k} \int_{t_{k-1}}^{t_{k}} q(s) e^{\int_{s}^{t_{k}} p(\tau) d \tau} d s\right. \\
& \left.\left.\left.+c_{k} \int_{t_{k}-\tau_{k}}^{t_{k}-\sigma_{k}} \int_{t_{k-1}}^{s} q(v) e^{\int_{v}^{s} p(\tau) d \tau} d v d s+b_{k}\right)\right]\right\} e^{\int_{t_{i}}^{t} p(\tau) d \tau} \\
& +\int_{t_{i}}^{t} q(s) e^{\int_{s}^{t} p(\tau) d \tau} d s, \quad t \geq t_{0} .
\end{aligned}
$$

This result can be used to investigate the qualitative properties of certain linear impulsive differential equations. 
A natural question arises, that is, how about the upper bound if the inequality is of nonlinearity? In this paper, under different jump conditions, we will study the upper-bound estimation of the nonlinear inequality

$$
m^{\prime}(t) \leq p(t) m(t)+q(t) m^{\alpha}(t)
$$

\section{Main results}

In this paper, let $0 \leq t_{0}<t_{1}<t_{2}<\cdots$ be a sequence. For $I \subset \mathbb{R}$, we denote by $\operatorname{PC}(I, \mathbb{R})$ the functions $u(t)$ defined on $I$, which is continuous for $t \neq t_{k}, u(0+), u\left(t_{k}+\right), u\left(t_{k}-\right)$ exist and $u(t)$ is left continuous at $t_{k}, k=1,2, \ldots, \mathrm{PC}^{1}\left(I, \mathbb{R}_{+}\right)$is the collection of functions $u(t)$ such that $u, u^{\prime} \in \operatorname{PC}\left(I, \mathbb{R}_{+}\right)$. Throughout this paper, we assume the following hypotheses:

$\left(\mathrm{H}_{0}\right)$ the sequence $\left\{t_{k}\right\}$ satisfies $0 \leq t_{0} \leq t_{1} \leq t_{2} \leq \cdots, \lim _{k \rightarrow \infty} t_{k}=+\infty$.

$\left(\mathrm{H}_{1}\right) \quad m \in \mathrm{PC}^{1}\left(I, \mathbb{R}_{+}\right)$, and $m(t)$ is left continuous at $t_{k}, k=1,2, \ldots$.

Lemma 2.1 (see [11]) Suppose that $a, b \in \mathbb{R}, p>0$. Then

$$
(|a|+|b|)^{p} \leq C_{p}\left(|a|^{p}+|b|^{p}\right)
$$

where $C_{p}=1$ for $0<p \leq 1$, and $C_{p}=2^{p-1}$ for $p>1$.

Theorem 2.1 Suppose that $\left(\mathrm{H}_{0}\right)$ and $\left(\mathrm{H}_{1}\right)$ hold. Iffor $k=1,2, \ldots, t \geq t_{0}$,

$$
\begin{aligned}
& m^{\prime}(t) \leq p(t) m(t)+q(t) m^{\alpha}(t), \quad t \neq t_{k}, \\
& m^{1-\alpha}\left(t_{k}^{+}\right) \leq d_{k} m^{1-\alpha}\left(t_{k}\right)+c_{k} \int_{t_{k}-\tau_{k}}^{t_{k}-\sigma_{k}} m^{1-\alpha}(s) d s+b_{k},
\end{aligned}
$$

here $0<\alpha<1, p, q \in C\left[\mathbb{R}_{+}, \mathbb{R}\right]$, and for $k=1,2, \ldots, t \geq t_{0}, c_{k} ; d_{k} \geq 0,0 \leq \sigma_{k} \leq \tau_{k} \leq t_{k}-t_{k-1}$, $b_{k}$ are constants. We have the estimation

$$
\begin{aligned}
m(t) \leq & \left\{\left[m^{1-\alpha}\left(t_{0}\right) \prod_{t_{0}<t_{k}<t} E_{k}+\sum_{t_{0}<t_{k}<t} G_{k} \prod_{t_{k}<t_{j}<t} E_{j}\right] e^{\int_{t_{i}}^{t}(1-\alpha) p(\tau) d \tau}\right. \\
& \left.+(1-\alpha) \int_{t_{i}}^{t} q(s) e^{\int_{s}^{t}(1-\alpha) p(\tau) d \tau} d s\right\}^{\frac{1}{1-\alpha}}, \quad t \geq t_{0},
\end{aligned}
$$

where

$$
\begin{aligned}
E_{k}= & d_{k} e^{\int_{t_{k-1}}^{t_{k}}(1-\alpha) p(\tau) d \tau}+c_{k} \int_{t_{k}-\tau_{k}}^{t_{k}-\sigma_{k}} e^{\int_{t_{k-1}}^{s}(1-\alpha) p(\tau) d \tau} d s, \\
G_{k}= & d_{k} \int_{t_{k-1}}^{t_{k}}(1-\alpha) q(s) e^{\int_{s}^{t_{k}}(1-\alpha) p(\tau) d \tau} d s \\
& +(1-\alpha) c_{k} \int_{t_{k}-\tau_{k}}^{t_{k}-\sigma_{k}} \int_{t_{k-1}}^{s} q(v) e^{\int_{v}^{s}(1-\alpha) p(\tau) d \tau} d v d s+b_{k} .
\end{aligned}
$$


Sha and Mend Advances in Difference Equations ( 2016) 2016:112

Page 4 of 13

Proof For $t \in\left[t_{0}, t_{1}\right]$, we have

$$
\frac{d}{d t}\left[e^{\int_{t_{0}}^{t}-(1-\alpha) p(\tau) d \tau} m^{1-\alpha}(t)\right] \leq(1-\alpha) q(t) e^{-\int_{t_{0}}^{t}(1-\alpha) p(\tau) d \tau}
$$

integrating (2.6) implies

$$
m^{1-\alpha}(t) \leq m^{1-\alpha}\left(t_{0}\right) e^{\int_{t_{0}}^{t}(1-\alpha) p(\tau) d \tau}+(1-\alpha) \int_{t_{0}}^{t} q(s) e^{\int_{s}^{t}(1-\alpha) p(\tau) d \tau} d s
$$

which shows that (2.3) holds for $t \in\left[t_{0}, t_{1}\right]$.

Now we suppose that (2.3) holds for $t \in\left[t_{0}, t_{n}\right]$, then we need only prove that (2.3) holds for $t \in\left(t_{n}, t_{n+1}\right]$ by mathematical induction. Since

$$
\begin{aligned}
& m^{1-\alpha}\left(t_{n}\right) \leq\left[m^{1-\alpha}\left(t_{0}\right) \prod_{t_{0}<t_{k}<t_{n}} E_{k}+\sum_{t_{0}<t_{k}<t_{n}} G_{k} \prod_{t_{k}<t_{j}<t_{n}} E_{j}\right] e^{\int_{t_{n-1}}^{t_{n}}(1-\alpha) p(\tau) d \tau} \\
& +(1-\alpha) \int_{t_{n-1}}^{t_{n}} q(s) e^{\int_{s}^{t_{n}}(1-\alpha) p(\tau) d \tau} d s \\
& =\left[m^{1-\alpha}\left(t_{0}\right) \prod_{i=1}^{n-1} E_{i}+\sum_{i=1}^{n-1} G_{i} \prod_{j=i+1}^{n-1} E_{j}\right] e^{\int_{t_{n-1}}^{t_{n}}(1-\alpha) p(\tau) d \tau} \\
& +(1-\alpha) \int_{t_{n-1}}^{t_{n}} q(s) e^{\int_{s}^{t_{n}}(1-\alpha) p(\tau) d \tau} d s \\
& m^{1-\alpha}\left(t_{n}^{+}\right) \leq d_{n} m^{1-\alpha}\left(t_{n}\right)+c_{n} \int_{t_{n}-\tau_{n}}^{t_{n}-\sigma_{n}} m^{1-\alpha}(s) d s+b_{n} \\
& \leq d_{n}\left\{\left[m^{1-\alpha}\left(t_{0}\right) \prod_{i=1}^{n-1} E_{i}+\sum_{i=1}^{n-1} G_{i} \prod_{j=i+1}^{n-1} E_{j}\right] e^{\int_{t_{n-1}}^{t_{n}}(1-\alpha) p(\tau) d \tau}\right. \\
& \left.+(1-\alpha) \int_{t_{n-1}}^{t_{n}} q(s) e^{\int_{s}^{t_{n}}(1-\alpha) p(\tau) d \tau} d s\right\} \\
& +c_{n} \int_{t_{n}-\tau_{n}}^{t_{n}-\sigma_{n}}\left[m^{1-\alpha}\left(t_{0}\right) \prod_{i=1}^{n-1} E_{i}+\sum_{i=1}^{n-1} G_{i} \prod_{j=i+1}^{n-1} E_{j}\right] e^{\int_{t_{n-1}}^{s}(1-\alpha) p(\tau) d \tau} d s \\
& +c_{n} \int_{t_{n}-\tau_{n}}^{t_{n}-\sigma_{n}}(1-\alpha) \int_{t_{n-1}}^{s} q(\nu) e^{\int_{v}^{s}(1-\alpha) p(\tau) d \tau} d \nu d s+b_{n} \\
& =\left[m^{1-\alpha}\left(t_{0}\right) \prod_{i=1}^{n-1} E_{i}+\sum_{i=1}^{n-1} G_{i} \prod_{j=i+1}^{n-1} E_{j}\right] \\
& \times\left[d_{n} e^{\int_{t_{n-1}}^{t_{n}}(1-\alpha) p(\tau) d \tau}+c_{n} \int_{t_{n}-\tau_{n}}^{t_{n}-\sigma_{n}} e^{\int_{t_{n-1}}^{s}(1-\alpha) p(\tau) d \tau} d s\right] \\
& +d_{n}(1-\alpha) \int_{t_{n-1}}^{t_{n}} q(s) e^{\int_{s}^{t_{n}} p(\tau) d \tau} d s \\
& +(1-\alpha) c_{n} \int_{t_{n}-\tau_{n}}^{t_{n}-\sigma_{n}} \int_{t_{n}-1}^{s} q(\nu) e^{\int_{v}^{s}(1-\alpha) p(\tau) d \tau} d \nu d s+b_{n} \\
& =\left[m^{1-\alpha}\left(t_{0}\right) \prod_{i=1}^{n-1} E_{i}+\sum_{i=1}^{n-1} G_{i} \prod_{j=i+1}^{n-1} E_{j}\right] E_{n}+G_{n}
\end{aligned}
$$




$$
\begin{aligned}
& =m^{1-\alpha}\left(t_{0}\right) \prod_{i=1}^{n} E_{i}+\left(\sum_{i=1}^{n-1} G_{i} \prod_{j=i+1}^{n-1} E_{j}\right) E_{n}+G_{n} \\
& =m^{1-\alpha}\left(t_{0}\right) \prod_{i=1}^{n} E_{i}+\sum_{i=1}^{n} G_{i} \prod_{j=i+1}^{n} E_{j}
\end{aligned}
$$

substituting (2.8) into (2.7), with $t_{0}$ being replaced by $t_{n}^{+}$, we obtain, for $t \in\left(t_{n}, t_{n+1}\right]$,

$$
\begin{aligned}
m^{1-\alpha}(t) \leq & m^{1-\alpha}\left(t_{n}^{+}\right) e^{\int_{t_{n}}^{t}(1-\alpha) p(\tau) d \tau}+(1-\alpha) \int_{t_{n}}^{t} q(s) e^{\int_{s}^{t}(1-\alpha) p(\tau) d \tau} d s \\
\leq & {\left[m^{1-\alpha}\left(t_{0}\right) \prod_{i=1}^{n} E_{i}+\sum_{i=1}^{n} G_{i} \prod_{j=i+1}^{n} E_{j}\right] e^{\int_{t_{n}}^{t}(1-\alpha) p(\tau) d \tau} } \\
& +(1-\alpha) \int_{t_{n}}^{t} q(s) e^{\int_{s}^{t}(1-\alpha) p(\tau) d \tau} d s \\
= & {\left[m^{1-\alpha}\left(t_{0}\right) \prod_{t_{0}<t_{k}<t_{n+1}} E_{k}+\sum_{t_{0}<t_{k}<t_{n+1}} G_{k} \prod_{t_{k}<t_{j}<t_{n+1}} E_{j}\right] e^{\int_{t_{n}}^{t}(1-\alpha) p(\tau) d \tau} } \\
& +(1-\alpha) \int_{t_{n}}^{t} q(s) e^{\int_{s}^{t}(1-\alpha) p(\tau) d \tau} d s .
\end{aligned}
$$

This completes the proof of Theorem 2.1.

If $d_{k} \equiv 0$ in Theorem 2.1, we obtain the following corollary.

Corollary 2.1 Suppose that $\left(\mathrm{H}_{0}\right)$ and $\left(\mathrm{H}_{1}\right)$ hold, $p, q \in C\left[\mathbb{R}_{+}, \mathbb{R}\right]$ and for $k=1,2, \ldots, t \geq t_{0}$,

$$
\begin{aligned}
& m^{\prime}(t) \leq p(t) m(t)+q(t) m^{\alpha}(t), \quad 0<\alpha<1, \\
& m^{1-\alpha}\left(t_{k}^{+}\right) \leq c_{k} \int_{t_{k}-\tau_{k}}^{t_{k}-\sigma_{k}} m^{1-\alpha}(s) d s+b_{k},
\end{aligned}
$$

where $c_{k}, b_{k}, \sigma_{k}, \tau_{k}$ are defined as in Theorem 2.1, then we have

$$
\begin{aligned}
m(t) \leq & \left\{\left[m^{1-\alpha}\left(t_{0}\right) \prod_{t_{0}<t_{k}<t} c_{k} \int_{t_{k}-\tau_{k}}^{t_{k}-\sigma_{k}} e^{\int_{t_{k-1}}^{s}(1-\alpha) p(\tau) d \tau} d s\right.\right. \\
& +\sum_{t_{0}<t_{k}<t}(1-\alpha) c_{k} \int_{t_{k}-\tau_{k}}^{t_{k}-\sigma_{k}} \int_{t_{k-1}}^{s} q(\nu) e^{\int_{v}^{s}(1-\alpha) p(\tau) d \tau} d \nu d s \\
& \left.\times \prod_{t_{k}<t_{j}<t} c_{j} \int_{t_{j}-\tau_{j}}^{t_{j}-\sigma_{j}} e^{\int_{t_{j-1}}^{s}(1-\alpha) p(\tau) d \tau} d s\right] e^{\int_{t_{i}}^{t}(1-\alpha) p(\tau) d \tau} \\
& \left.+(1-\alpha) \int_{t_{i}-1}^{t} q(s) e^{\int_{s}^{t}(1-\alpha) p(\tau) d \tau} d s\right\}^{\frac{1}{1-\alpha}} .
\end{aligned}
$$

If $d_{k} \equiv 1$, we obtain the following theorem. 
Theorem 2.2 Suppose that $\left(\mathrm{H}_{0}\right)$ and $\left(\mathrm{H}_{1}\right)$ hold. If, for $k=1,2, \ldots, t \geq t_{0}$,

$$
\left\{\begin{array}{l}
m^{\prime}(t) \leq p(t) m(t)+q(t) m^{\alpha}(t), \quad t \neq t_{k} \\
\Delta m^{1-\alpha}\left(t_{k}\right) \leq c_{k} \int_{t_{k}-\tau_{k}}^{t_{k}-\sigma_{k}} m^{1-\alpha}(s) d s+b_{k}
\end{array}\right.
$$

where $0<\alpha<1, p, q \in C\left[\mathbb{R}_{+}, \mathbb{R}\right]$, and for $k=1,2, \ldots, t \geq t_{0}, c_{k} \geq 0,0 \leq \sigma_{k} \leq \tau_{k} \leq t_{k}-t_{k-1}$, $b_{k}$ are constants, $\Delta m^{1-\alpha}\left(t_{k}\right)=m^{1-\alpha}\left(t_{k}^{+}\right)-m^{1-\alpha}\left(t_{k}\right)$. We have the estimation

$$
\begin{aligned}
m(t) \leq & \left\{\left[m^{1-\alpha}\left(t_{0}\right) \prod_{t_{0}<t_{k}<t} E_{k}+\sum_{t_{0}<t_{k}<t} H_{k} \prod_{t_{k}<t_{j}<t} E_{j}\right] e^{\int_{t_{i}}^{t}(1-\alpha) p(\tau) d \tau}\right. \\
& \left.+(1-\alpha) \int_{t_{0}}^{t} q(s) e^{\int_{s}^{t}(1-\alpha) p(\tau) d \tau} d s\right\}^{\frac{1}{1-\alpha}}, \quad t \geq t_{0}
\end{aligned}
$$

where $E_{k}$ is defined as (2.4) (with $\left.d_{k} \equiv 1\right)$,

$$
H_{k}=(1-\alpha) c_{k} \int_{t_{k}-\tau_{k}}^{t_{k}-\sigma_{k}} \int_{t_{0}}^{s} q(\nu) e^{\int_{v}^{s}(1-\alpha) p(\tau) d \tau} d \nu d s+b_{k}
$$

Proof As the proof of Theorem 2.1, we prove (2.7) holds for $t \in\left[t_{0}, t_{1}\right]$, which means that (2.10) holds for $t \in\left[t_{0}, t_{1}\right]$. Now suppose that (2.10) holds for $t \in\left[t_{0}, t_{n}\right]$, then

$$
\begin{aligned}
m^{1-\alpha}\left(t_{n}\right) \leq & {\left[m^{1-\alpha}\left(t_{0}\right) \prod_{t_{0}<t_{k}<t} E_{k}+\sum_{t_{0}<t_{k}<t} H_{k} \prod_{t_{k}<t_{j}<t} E_{j}\right] e^{\int_{t_{n-1}}^{t_{n}}(1-\alpha) p(\tau) d \tau} } \\
& +(1-\alpha) \int_{t_{0}}^{t_{n}} q(s) e^{\int_{s}^{t_{n}}(1-\alpha) p(\tau) d \tau} d s \\
= & {\left[m^{1-\alpha}\left(t_{0}\right) \prod_{i=1}^{n-1} E_{i}+\sum_{i=1}^{n-1} H_{i} \prod_{j=i+1}^{n-1} E_{j}\right] e^{\int_{t_{n-1}}^{t_{n}}(1-\alpha) p(\tau) d \tau} } \\
& +(1-\alpha) \int_{t_{0}}^{t_{n}} q(s) e^{\int_{s}^{t_{n}}(1-\alpha) p(\tau) d \tau} d s .
\end{aligned}
$$

So

$$
\begin{aligned}
m^{1-\alpha}\left(t_{n}^{+}\right) \leq & m^{1-\alpha}\left(t_{n}\right)+c_{n} \int_{t_{n}-\tau_{n}}^{t_{n}-\sigma_{n}} m^{1-\alpha}(s) d s+b_{n} \\
\leq & {\left[m^{1-\alpha}\left(t_{0}\right) \prod_{i=1}^{n-1} E_{i}+\sum_{i=1}^{n-1} H_{i} \prod_{j=i+1}^{n-1} E_{j}\right] e^{\int_{t_{n-1}}^{t_{n}}(1-\alpha) p(\tau) d \tau} } \\
& +(1-\alpha) \int_{t_{0}}^{t_{n}} q(s) e^{\int_{s}^{t_{n}}(1-\alpha) p(\tau) d \tau} d s \\
& +c_{n} \int_{t_{n}-\tau_{n}}^{t_{n}-\sigma_{n}}\left[m^{1-\alpha}\left(t_{0}\right) \prod_{i=1}^{n-1} E_{i}+\sum_{i=1}^{n-1} H_{i} \prod_{j=i+1}^{n-1} E_{j}\right] e^{\int_{t_{n-1}}^{s}(1-\alpha) p(\tau) d \tau} d s \\
& +c_{n} \int_{t_{n}-\tau_{n}}^{t_{n}-\sigma_{n}}(1-\alpha) \int_{t_{0}}^{s} q(\nu) e^{\int_{v}^{s}(1-\alpha) p(\tau) d \tau} d \nu d s+b_{n}
\end{aligned}
$$




$$
\begin{aligned}
= & {\left[m^{1-\alpha}\left(t_{0}\right) \prod_{i=1}^{n-1} E_{i}+\sum_{i=1}^{n-1} H_{i} \prod_{j=i+1}^{n-1} E_{j}\right]\left[e^{\int_{t_{n-1}}^{t_{n}}(1-\alpha) p(\tau) d \tau}\right.} \\
& \left.+c_{n} \int_{t_{n}-\tau_{n}}^{t_{n}-\sigma_{n}} e^{\int_{t_{n-1}}^{s}(1-\alpha) p(\tau) d \tau} d s\right] \\
& +(1-\alpha) \int_{t_{0}}^{t_{n}} q(s) e^{\int_{s}^{t_{n}}(1-\alpha) p(\tau) d \tau} d s+H_{n} \\
= & m^{1-\alpha}\left(t_{0}\right) \prod_{i=1}^{n} E_{i}+\left(\sum_{i=1}^{n-1} H_{i} \prod_{j=i+1}^{n-1} E_{j}\right) E_{n} \\
& +H_{n}+(1-\alpha) \int_{t_{0}}^{t_{n}} q(s) e^{\int_{s}^{t_{n}}(1-\alpha) p(\tau) d \tau} d s \\
= & m^{1-\alpha}\left(t_{0}\right) \prod_{i=1}^{n} E_{i}+\sum_{i=1}^{n} H_{i} \prod_{j=i+1}^{n} E_{j} \\
& +(1-\alpha) \int_{t_{0}}^{t_{n}} q(s) e^{\int_{s}^{t_{n}}(1-\alpha) p(\tau) d \tau} d s .
\end{aligned}
$$

Using (2.7) (with $t_{0}$ being replaced by $\left.t_{n}^{+}\right)$, we obtain, for $t \in\left(t_{n}, t_{n+1}\right]$,

$$
\begin{aligned}
m^{1-\alpha}(t) \leq & {\left[m^{1-\alpha}\left(t_{0}\right) \prod_{i=1}^{n} E_{i}+\sum_{i=1}^{n} H_{i} \prod_{j=i+1}^{n} E_{j}+(1-\alpha) \int_{t_{0}}^{t_{n}} q(s) e^{\int_{s}^{t_{n}}(1-\alpha) p(\tau) d \tau} d s\right] } \\
& \times e^{\int_{t_{n}}^{t}(1-\alpha) p(\tau) d \tau}+(1-\alpha) \int_{t_{n}}^{t} q(s) e^{\int_{s}^{t}(1-\alpha) p(\tau) d \tau} d s \\
= & {\left[m^{1-\alpha}\left(t_{0}\right) \prod_{i=1}^{n} E_{i}+\sum_{i=1}^{n} H_{i} \prod_{j=i+1}^{n} E_{j}\right] e^{\int_{t_{n}}^{t}(1-\alpha) p(\tau) d \tau} } \\
& +(1-\alpha) \int_{t_{0}}^{t} q(s) e^{\int_{s}^{t}(1-\alpha) p(\tau) d \tau} d s \\
= & {\left[m^{1-\alpha}\left(t_{0}\right) \prod_{t_{0}<t_{k}<t_{n+1}} E_{k}+\sum_{t_{0}<t_{k}<t_{n+1}} H_{k} \prod_{t_{k}<t_{j}<t_{n+1}} E_{j}\right] e^{\int_{t_{n}}^{t}(1-\alpha) p(\tau) d \tau} } \\
& +(1-\alpha) \int_{t_{0}}^{t} q(s) e^{\int_{s}^{t}(1-\alpha) p(\tau) d \tau} d s .
\end{aligned}
$$

This completes the proof.

Remark 2.1 If $\alpha=0$, then Theorem 2.1 reduces to Theorem 1.1, and Theorem $2.2 \mathrm{im}$ proves Theorem 1.1.

If $p(t) \equiv 0$ in Theorem 2.2, we obtain the following useful corollary.

Corollary 2.2 If $\left(\mathrm{H}_{0}\right)$ and $\left(\mathrm{H}_{1}\right)$ hold and for $k=1,2, \ldots, t \geq t_{0}$,

$$
\left\{\begin{array}{l}
m^{\prime}(t) \leq q(t) m^{\alpha}(t), \\
\Delta m^{1-\alpha}(t) \leq c_{k} \int_{t_{k}-\tau_{k}}^{t_{k}-\tau_{k}} m^{1-\alpha}(s) d s+b_{k},
\end{array}\right.
$$


then

$$
\begin{aligned}
m(t) \leq & \left\{m^{1-\alpha}\left(t_{0}\right) \prod_{t_{0}<t_{k}<t}\left(1+c_{k}\left(\tau_{k}-\sigma_{k}\right)\right)\right. \\
& +\sum_{t_{0}<t_{k}<t}\left[(1-\alpha) c_{k}\left(\int_{t_{k}-\tau_{k}}^{t_{k}-\sigma_{k}} \int_{t_{k-1}}^{s} q(v) d v d s+b_{k}\right) \prod_{t_{k}<t_{j}<t}\left(1+c_{j}\left(\tau_{j}-\sigma_{j}\right)\right)\right] \\
& \left.+(1-\alpha) \int_{t_{0}}^{t} q(s) d s\right\}^{\frac{1}{1-\alpha}} .
\end{aligned}
$$

Next, we will give another kind of nonlinear impulsive differential inequalities.

Theorem 2.3 Suppose that $\left(\mathrm{H}_{0}\right)$ holds, and $m \in \mathrm{PC}^{1}\left[\mathbb{R}_{+}, \mathbb{R}_{+}\right], m(t)$ is left continuous at $t_{k}$, $k=1,2, \ldots, p(t), q(t) \in C\left[\mathbb{R}_{+}, \mathbb{R}_{+}\right]$. Assume

$$
\left\{\begin{array}{l}
m^{\prime}(t) \leq p(t) m(t)+q(t) m^{\alpha}(t), \quad t \neq t_{k} \\
\Delta m\left(t_{k}\right) \leq c_{k} \int_{t_{k}-\tau_{k}}^{t_{k}-\sigma_{k}} m(s) d s+b_{k}
\end{array}\right.
$$

where $\Delta m\left(t_{k}\right)=m\left(t_{k}^{+}\right)-m\left(t_{k}\right), 0<\alpha<1, c_{k} \geq 0,0 \leq \sigma_{k} \leq \tau_{k} \leq t_{k}-t_{k-1}, b_{k}$ are constants. We have the estimation

$$
\begin{aligned}
m^{1-\alpha}(t) \leq & \left(m\left(t_{0}\right) \prod_{t_{0}<t_{k}<t} F_{k}+\sum_{t_{0}<t_{k}<t} R_{k} \prod_{t_{k}<t_{j}<t} F_{j}\right)^{1-\alpha} e^{\int_{t_{i}}^{t}(1-\alpha) p(\tau) d \tau} \\
& +2^{(k-1) \alpha}(1-\alpha) \int_{t_{0}}^{t} q(s) e^{\int_{s}^{t}(1-\alpha) p(\tau) d \tau} d s, \quad t \geq t_{0}
\end{aligned}
$$

where

$$
\begin{aligned}
& F_{k}=2^{\frac{\alpha}{1-\alpha}}\left[e^{\int_{t_{k-1}}^{t_{k}} p(\tau) d \tau}+c_{k} \int_{t_{k}-\tau_{k}}^{t_{k}-\sigma_{k}} e^{\int_{t_{k-1}}^{s} p(\tau) d \tau} d s\right], \\
& R_{k}=c_{k} 2^{\frac{(k-1) \alpha}{1-\alpha}}(1-\alpha)^{\frac{1}{1-\alpha}} \int_{t_{k}-\tau_{k}}^{t_{k}-\sigma_{k}}\left(\int_{t_{0}}^{v} q(s) e^{\int_{s}^{v}(1-\alpha) p(\tau) d \tau} d s\right)^{\frac{1}{1-\alpha}} d v+b_{k} .
\end{aligned}
$$

Proof Obviously, (2.13) holds for $t \in\left[t_{0}, t_{1}\right]$ as (2.7). Now we suppose (2.13) holds for $t \in$ $\left[t_{0}, t_{n}\right]$, then by mathematical induction, we see that

$$
\begin{aligned}
m^{1-\alpha}\left(t_{n}\right) \leq & \left(m\left(t_{0}\right) \prod_{t_{0}<t_{k}<t_{n}} F_{k}+\sum_{t_{0}<t_{k}<t_{n}} R_{k} \prod_{t_{k}<t_{j}<t_{n}} F_{j}\right)^{1-\alpha} e^{\int_{t_{n-1}}^{t_{n}}(1-\alpha) p(\tau) d \tau} \\
& +2^{(n-1) \alpha}(1-\alpha) \int_{t_{0}}^{t_{n}} q(s) e^{\int_{s}^{t_{n}}(1-\alpha) p(\tau) d \tau} d s \\
= & \left(m\left(t_{0}\right) \prod_{i=1}^{n-1} F_{i}+\sum_{i=1}^{n-1} R_{i} \prod_{j=i+1}^{n-1} F_{j}\right)^{1-\alpha} e^{\int_{t_{n-1}}^{t_{n}}(1-\alpha) p(\tau) d \tau} \\
& +2^{(n-1) \alpha}(1-\alpha) \int_{t_{0}}^{t_{n}} q(s) e^{\int_{s}^{t_{n}}(1-\alpha) p(\tau) d \tau} d s .
\end{aligned}
$$


Sha and Mend Advances in Difference Equations ( 2016) 2016:112

Page 9 of 13

Since $\frac{1}{1-\alpha}>1$, by Lemma 2.1 ,

$$
\begin{aligned}
& m\left(t_{n}\right) \leq 2^{\frac{\alpha}{1-\alpha}}\left(m\left(t_{0}\right) \prod_{i=1}^{n-1} F_{i}+\sum_{i=1}^{n-1} R_{i} \prod_{j=i+1}^{n-1} F_{j}\right) e^{\int_{t_{n-1}}^{t_{n}} p(\tau) d \tau} \\
& +2^{\frac{\alpha}{1-\alpha}} 2^{\frac{(n-1) \alpha}{1-\alpha}}(1-\alpha)^{\frac{1}{1-\alpha}}\left(\int_{t_{0}}^{t_{n}} q(s) e^{\int_{s}^{t_{n}}(1-\alpha) p(\tau) d \tau} d s\right)^{\frac{1}{1-\alpha}}, \\
& m\left(t_{n}^{+}\right) \leq m\left(t_{n}\right)+c_{n} \int_{t_{n}-\tau_{n}}^{t_{n}-\sigma_{n}} m(s) d s+b_{n} \\
& \leq 2^{\frac{\alpha}{1-\alpha}}\left(m\left(t_{0}\right) \prod_{i=1}^{n-1} F_{i}+\sum_{i=1}^{n-1} R_{i} \prod_{j=i+1}^{n-1} F_{j}\right) e^{\int_{t_{n-1}}^{t_{n}} p(\tau) d \tau} \\
& +2^{\frac{n \alpha}{1-\alpha}}(1-\alpha)^{\frac{1}{1-\alpha}}\left(\int_{t_{0}}^{t_{n}} q(s) e^{\int_{s}^{t_{n}}(1-\alpha) p(\tau) d \tau} d s\right)^{\frac{1}{1-\alpha}} \\
& +c_{n} \int_{t_{n}-\tau_{n}}^{t_{n}-\sigma_{n}} 2 \frac{\alpha}{1-\alpha}\left(m\left(t_{0}\right) \prod_{i=1}^{n-1} F_{i}+\sum_{i=1}^{n-1} R_{i} \prod_{j=i+1}^{n-1} F_{j}\right) e^{\int_{t_{n-1}}^{s} p(\tau) d \tau} d s \\
& +c_{n} 2^{\frac{n \alpha}{1-\alpha}}(1-\alpha)^{\frac{1}{1-\alpha}} \int_{t_{n}-\tau_{n}}^{t_{n}-\sigma_{n}}\left(\int_{t_{0}}^{v} q(s) e^{\int_{s}^{v}(1-\alpha) p(\tau) d \tau} d s\right)^{\frac{1}{1-\alpha}} d \nu+b_{n} \\
& =\left(m\left(t_{0}\right) \prod_{i=1}^{n-1} F_{i}+\sum_{i=1}^{n-1} R_{i} \prod_{j=i+1}^{n-1} F_{j}\right) \\
& \times\left[2^{\frac{\alpha}{1-\alpha}}\left(e^{\int_{t_{n-1}}^{t_{n}} p(\tau) d \tau}+c_{n} \int_{t_{n}-\tau_{n}}^{t_{n}-\sigma_{n}} e^{\int_{t_{n-1}}^{s} p(\tau) d \tau} d s\right)\right] \\
& +R_{n}+2^{\frac{n \alpha}{1-\alpha}}(1-\alpha)^{\frac{1}{1-\alpha}}\left(\int_{t_{0}}^{t_{n}} q(s) e^{\int_{s}^{t_{n}}(1-\alpha) p(\tau) d \tau} d s\right)^{\frac{1}{1-\alpha}} \\
& =\left(m\left(t_{0}\right) \prod_{i=1}^{n-1} F_{i}+\sum_{i=1}^{n-1} R_{i} \prod_{j=i+1}^{n-1} F_{j}\right) F_{n}+R_{n} \\
& +2^{\frac{n \alpha}{1-\alpha}}(1-\alpha)^{\frac{1}{1-\alpha}}\left(\int_{t_{0}}^{t_{n}} q(s) e^{\int_{s}^{t_{n}}(1-\alpha) p(\tau) d \tau} d s\right)^{\frac{1}{1-\alpha}} \\
& =m\left(t_{0}\right) \prod_{i=1}^{n} F_{i}+\sum_{i=1}^{n} R_{i} \prod_{j=i+1}^{n} F_{j} \\
& +2^{\frac{n \alpha}{1-\alpha}}(1-\alpha)^{\frac{1}{1-\alpha}}\left(\int_{t_{0}}^{t_{n}} q(s) e^{\int_{s}^{t_{n}}(1-\alpha) p(\tau) d \tau} d s\right)^{\frac{1}{1-\alpha}} .
\end{aligned}
$$

So for $t \in\left(t_{n}, t_{n+1}\right]$, since $0<1-\alpha<1$, by Lemma 2.1 and (2.7) (with $t_{0}$ being replaced by $\left.t_{n}^{+}\right)$, we obtain

$$
\begin{aligned}
m^{1-\alpha}(t) \leq & m^{1-\alpha}\left(t_{n}^{+}\right) e^{\int_{t_{n}}^{t}(1-\alpha) p(\tau) d \tau} \\
& +(1-\alpha) \int_{t_{n}}^{t} q(s) e^{\int_{s}^{t}(1-\alpha) p(\tau) d \tau} d s
\end{aligned}
$$




$$
\begin{aligned}
= & {\left[m\left(t_{0}\right) \prod_{i=1}^{n} F_{i}+\sum_{i=1}^{n} R_{i} \prod_{j=i+1}^{n} F_{j}\right.} \\
& \left.+2^{\frac{n \alpha}{1-\alpha}}(1-\alpha)^{\frac{1}{1-\alpha}}\left(\int_{t_{0}}^{t_{n}} q(s) e^{\int_{s}^{t_{n}}(1-\alpha) p(\tau) d \tau} d s\right)^{\frac{1}{1-\alpha}}\right]^{1-\alpha} \\
& \times e^{\int_{t_{n}}^{t}(1-\alpha) p(\tau) d \tau}+(1-\alpha) \int_{t_{n}}^{t} q(s) e^{\int_{s}^{t}(1-\alpha) p(\tau) d \tau} d s \\
\leq & {\left[\left(m\left(t_{0}\right) \prod_{i=1}^{n} F_{i}+\sum_{i=1}^{n} R_{i} \prod_{j=i+1}^{n} F_{j}\right)^{1-\alpha}\right.} \\
& \left.+2^{n \alpha}(1-\alpha) \int_{t_{0}}^{t_{n}} q(s) e^{\int_{s}^{t_{n}}(1-\alpha) p(\tau) d \tau} d s\right] \\
& \times e^{\int_{t_{n}}^{t}(1-\alpha) p(\tau) d \tau}+(1-\alpha) \int_{t_{n}}^{t} q(s) e^{\int_{s}^{t}(1-\alpha) p(\tau) d \tau} d s \\
\leq & \left(m\left(t_{0}\right) \prod_{i=1}^{n} F_{i}+\sum_{i=1}^{n} R_{i} \prod_{j=i+1}^{n} F_{j}\right)^{1-\alpha} e^{\int_{t_{n}}^{t}(1-\alpha) p(\tau) d \tau} \\
& +2^{n \alpha}(1-\alpha) \int_{t_{0}}^{t} q(s) e^{\int_{s}^{t}(1-\alpha) p(\tau) d \tau} d s \\
= & \left(m\left(t_{0}\right) \prod_{t_{0}<t_{k}<t_{n+1}} F_{k}+\sum_{t_{0}<t_{k}<t_{n+1}} R_{k} \prod_{t_{k}<t_{j}<t_{n+1}} F_{j}\right)^{1-\alpha} e^{\int_{t_{n}}^{t}(1-\alpha) p(\tau) d \tau} \\
& +2^{n \alpha}(1-\alpha) \int_{t_{0}}^{t} q(s) e^{\int_{s}^{t}(1-\alpha) p(\tau) d \tau} d s,
\end{aligned}
$$

which shows that (2.13) holds for $k=n+1$. This completes the proof.

Now we give an upper-bound estimation of a nonlinear integral inequality with integral jump conditions.

Theorem 2.4 Suppose that $\left(\mathrm{H}_{0}\right)$ holds, and suppose $m, p, q \in C\left[\mathbb{R}_{+}, \mathbb{R}_{+}\right]$. For $t \geq t_{0}$, if

$$
m(t) \leq c+\int_{t_{0}}^{t} p(s) m(s) d s+\int_{t_{0}}^{t} q(s) m^{\alpha}(s) d s+\sum_{t_{0}<t_{k}<t} \alpha_{k} \int_{t_{k}-\tau_{k}}^{t_{k}-\sigma_{k}} m(s) d s
$$

where $\alpha_{k} \geq 0,0 \leq \sigma_{k} \leq \tau_{k} \leq t_{k}-t_{k-1}, c \geq 0,0<\alpha<1$ are constants. Then we have the estimation

$$
\begin{aligned}
m(t) \leq & \left\{\left(c \prod_{t_{0}<t_{k}<t} F_{k}+\sum_{t_{0}<t_{k}<t} R_{k} \prod_{t_{k}<t_{j}<t} F_{j}\right)^{1-\alpha} e^{\int_{t_{i}}^{t}(1-\alpha) p(\tau) d \tau}\right. \\
& \left.+2^{(k-1) \alpha}(1-\alpha) \int_{t_{0}}^{t} q(s) e^{\int_{s}^{t}(1-\alpha) p(\tau) d \tau} d s\right\}^{1 /(1-\alpha)}
\end{aligned}
$$

where $F_{k}$ and $R_{k}$ are defined as that in Theorem 2.3, with $c_{k}$ being replaced by $\alpha_{k}$. 
Proof Defined the right-hand side of (2.14) as a new function $v(t)$, we have $m(t) \leq v(t)$ and $v\left(t_{0}\right)=c$. Since

$$
\begin{aligned}
& v^{\prime}(t)=p(t) m(t)+q(t) m^{\alpha}(t), \quad t \neq t_{k}, \\
& v\left(t_{k}+\right)=v\left(t_{k}\right)+\alpha_{k} \int_{t_{k}-\tau_{k}}^{t_{k}-\sigma_{k}} m(s) d s,
\end{aligned}
$$

we obtain further

$$
\begin{aligned}
& v^{\prime}(t) \leq p(t) v(t)+q(t) v^{\alpha}(t), \quad t \neq t_{k}, \\
& v\left(t_{k}+\right)=v\left(t_{k}\right)+\alpha_{k} \int_{t_{k}-\tau_{k}}^{t_{k}-\sigma_{k}} v(s) d s .
\end{aligned}
$$

Then using Theorem 2.3 implies the estimation of $v(t)$, the estimation of the unknown function $m(t)$ is obtained since $m(t) \leq v(t)$, and this completes the proof.

\section{Application to impulsive differential equations}

As an application of Theorem 2.4, we give an upper-bound estimation of certain nonlinear impulsive differential equation as follows:

$$
\left\{\begin{array}{l}
v^{\prime}(t)=f(t, v), \quad t \neq t_{k}, \\
\Delta v\left(t_{k}\right)=I_{k}\left(\int_{t_{k}-\tau_{k}}^{t_{k}-\sigma_{k}} v(s) d s\right), \quad t \in\left[t_{0}, \infty\right), \\
v\left(t_{0}\right)=v_{0},
\end{array}\right.
$$

where $f \in C(\mathbb{R} \times \mathbb{R}, \mathbb{R}), I_{k} \in C(\mathbb{R}, \mathbb{R}), 0<t_{0}<t_{1}<\cdots, \lim _{t \rightarrow \infty} t_{k}=+\infty, 0 \leq \sigma_{k} \leq \tau_{k} \leq$ $t_{k}-t_{k-1}, k=1,2, \ldots$. If there exists $L>0$ such that

$$
|f(t, v)| \leq L|v|^{\alpha}, \quad 0<\alpha<1
$$

and there exist $\iota_{k} \geq 0$, such that

$$
\left|I_{k}(v)\right| \leq \iota_{k}|v|, \quad k=1,2, \ldots,
$$

then for any solution $v(t)$ of (3.1), we have

$$
\begin{aligned}
|v(t)| \leq & \left\{\left(\left|v_{0}\right| \prod_{t_{0}<t_{k}<t} 2^{\alpha /(1-\alpha)}\left(1+\iota_{k}\left(\tau_{k}-\sigma_{k}\right)\right)\right.\right. \\
& +\sum_{t_{0}<t_{k}<t} 2^{\frac{(k-1) \alpha}{1-\alpha}}(L(1-\alpha))^{\frac{1}{1-\alpha}} \iota_{k}\left(t_{k}-t_{0}\right)^{\frac{2-\alpha}{1-\alpha}} \\
& \left.\times \prod_{t_{k}<t_{j}<t} 2^{\frac{\alpha}{1-\alpha}}\left(1+\iota_{j}\left(\tau_{j}-\sigma_{j}\right)\right)\right)^{1-\alpha} \\
& \left.+2^{(k-1) \alpha}(1-\alpha) L\left(t-t_{0}\right)\right\}^{1 /(1-\alpha)}
\end{aligned}
$$


Proof Suppose $v=v(t)$ is a solution of (3.1), we integrate (3.1) to obtain

$$
\begin{aligned}
v(t) & =v\left(t_{0}\right)+\int_{t_{0}}^{t} f(s, v(s)) d s+\sum_{t_{0}<t_{k}<t} I_{k}\left(\int_{t_{k}-\tau_{k}}^{t_{k}-\sigma_{k}} v(s) d s\right) \\
& =v_{0}+\int_{t_{0}}^{t} f(s, v(s)) d s+\sum_{t_{0}<t_{k}<t} I_{k}\left(\int_{t_{k}-\tau_{k}}^{t_{k}-\sigma_{k}} v(s) d s\right) .
\end{aligned}
$$

By (3.2) and (3.3), we obtain

$$
\begin{aligned}
|v(t)| & \leq\left|v_{0}\right|+\int_{t_{0}}^{t}|f(s, v(s))| d s+\sum_{t_{0}<t_{k}<t}\left|I_{k}\left(\int_{t_{k}-\tau_{k}}^{t_{k}-\sigma_{k}} v(s) d s\right)\right| \\
& \leq\left|v_{0}\right|+L \int_{t_{0}}^{t}|v(s)|^{\alpha} d s+\sum_{t_{0}<t_{k}<t} \iota_{k} \int_{t_{k}-\tau_{k}}^{t_{k}-\sigma_{k}}|v(s)| d s .
\end{aligned}
$$

Then by Theorem 2.4, we compute that

$$
\begin{aligned}
F_{k} & =2^{\alpha /(1-\alpha)}\left(1+\iota_{k}\left(\tau_{k}-\sigma_{k}\right)\right) ; \\
R_{k} & =\iota_{k} 2^{\frac{(k-1) \alpha}{1-\alpha}}(1-\alpha)^{\frac{1}{1-\alpha}} \int_{t_{k}-\tau_{k}}^{t_{k}-\sigma_{k}}\left(\int_{t_{0}}^{v} L d s\right)^{1 /(1-\alpha)} d v \\
& =\frac{1-\alpha}{2-\alpha} 2^{\left(\frac{(k-1) \alpha}{1-\alpha}\right.}(L(1-\alpha))^{\frac{1}{1-\alpha}} \iota_{k}\left[\left(t_{k}-\sigma_{k}-t_{0}\right)^{\frac{2-\alpha}{1-\alpha}}-\left(t_{k}-\tau_{k}-t_{0}\right)^{\frac{2-\alpha}{1-\alpha}}\right] \\
& \leq 2^{\frac{(k-1) \alpha}{1-\alpha}}(L(1-\alpha))^{\frac{1}{1-\alpha}} \iota_{k}\left(t_{k}-t_{0}\right)^{\frac{2-\alpha}{1-\alpha}} .
\end{aligned}
$$

Substituting $F_{k}$ and $R_{k}$ in (2.15), we obtain (3.4). This completes the proof.

\section{Competing interests}

The authors declare that there are no competing interests.

\section{Authors' contributions}

JS gave the main theorems; FM gave some useful comments and revised the paper. All authors have read and approved the final manuscript.

\section{Author details}

'Department of Mathematics, Jining University, Qufu, Shandong 273155, P.R. China. ${ }^{2}$ School of Mathematical Sciences, Qufu Normal University, Qufu, Shandong 273155, P.R. China.

\section{Acknowledgements}

The authors sincerely thank the referees for their constructive suggestions and corrections. This research was partially supported by the NSF of Shandong Province (Grant ZR2015PA005), the NNSF of China (Grant 11271225).

Received: 13 January 2016 Accepted: 11 April 2016 Published online: 22 April 2016

\section{References}

1. Lakshmikantham, V, Bainov, DD, Simeonov, PS: Theory of Impulsive Differential Equations. World Scientific, Singapore (1989)

2. Bainov, DD, Simeonov, PS: Impulsive Differential Equations: Periodic Solutions and Applications. Longman, Harlow (1993)

3. Bainov, DD, Simeonov, PS: Impulsive Differential Equations: Asymptotic Properties of the Solutions. World Scientific, Singapore (1995)

4. Samoilenko, AM, Perestyuk, NA: Impulsive Differential Equations. World Scientific, Singapore (1995)

5. Wang, W-S: A generalized Gronwall-Bellman integral inequality with impulsive function and its application. J. Sichuan Normal Univ. (Nat. Sci.) 34(1), 43-46 (2011)

6. Deng, S, Prather, C: Generalization of an impulsive nonlinear singular Gronwall-Bihari inequality with delay. J. Inequal. Pure Appl. Math. 9(2), 34 (2008). 
7. Hristova, SG: Nonlinear delay integral inequalities for piecewise continuous functions and applications. J. Inequal. Pure Appl. Math. 5(4), 88 (2004)

8. Li, J: On some new impulsive integral inequalities. J. Inequal. Appl. 28, 312395 (2008). doi:10.1155/2008/312395312-395

9. Tatar, NE: An impulsive nonlinear singular version of the Gronwall-Bihari inequality. J. Inequal. Appl. 2006, 84561 (2006). doi:10.1155/JIA/2006/84561

10. Wang, H, Ding, C: A new nonlinear impulsive delay differential inequality and its applications. J. Inequal. Appl. 2011, 11 (2011). doi:10.1186/1029-242X-2011-11

11. Garling, DJH: Inequalities, a Journey into Linear Analysis. Cambridge University Press, Cambridge (2007)

12. Zheng, Z, Gao, X, Shao, J: Some new generalized retarded inequalities for discontinuous functions and their applications. J. Inequal. Appl. 2016, 7 (2016). doi:10.1186/s13660-015-0943-6

13. Borysenko, DS: About one integral inequality for piece-wise continuous functions. In: Proceedings of 10th International Kravchuk Conference, Kyiv (2004), p. 323

14. Iovane, G: Some new integral inequalities of Bellman-Bihari type with delay for discontinuous functions. Nonlinear Anal. 66(2), 498-508 (2007)

15. Thiramanus, $\mathrm{P}$, Tariboon, J: Impulsive differential and impulsive integral inequalities with integral jump conditions. J. Inequal. Appl. 2012, 25 (2012). doi:10.1186/1029-242X-2012-25

\section{Submit your manuscript to a SpringerOpen ${ }^{\circ}$ journal and benefit from:}

- Convenient online submission

- Rigorous peer review

- Immediate publication on acceptance

- Open access: articles freely available online

- High visibility within the field

- Retaining the copyright to your article 